\title{
Mixing and Recirculation Characteristics of A Double Concentric Burner with Bluff-Body
}

\author{
H.K. Ma C.H. Chiou J.S. Harn \\ Department of Mechanical Engineering, National Taiwan University, Taipei, Taiwan, China
}

\begin{abstract}
The concentric bluff-body jet burner is widely used in industrial combustion systems. This kind of burner often generates a considerably complex recirculation zone behind the bluff body. As a result, the fuel often remains in the recirculation zone, achieving stability of flame. This study investigates, by means of experiments, the variations of the aerodynamics as the fluid is injected into a combustion chamber through a double concentric burner with a bluff-body. The observation and measurement of the aerodynamics in our experiment are conducted under a cold flow. The controlled parameters in our experiment are: variations in the blockage ratio of the center bluff body, the cone angle of the bluff body, and the velocity ratio $\left(U_{s} / U_{p}\right)$ of the secondary jet and primary jet; the injection of helium bubbles into the primary and secondary jets to observe the recirculation zone behind the bluff body; using Tufts for observing the characteristics of corner recirculation zone in a combustion chamber, measuring the average velocity of each point. within the aerodynamics by the 5 hole pitot tube; measuring the clistribution of static pressure of the combustion chamber walls with a static pressure tap.
\end{abstract}

Keywords: concentric jet burner with bluff body, internal aerodynamics of the burner, parameters affecting the flow fields in the burner.

\section{INTRODUCTION}

The flow field of the double concentric bluff-body jet as it enters the combustion chamber primarily consists of the principal circulation zone created by the primary and secondary jets, the recirculation zone behind the bluff body, and the outer recirculation zone formed by the projecting walls. This recirculation zone forms a low velocity region with longer residence time, in which the ignition source may linger and transmit the flame over to the unburned mixture, generating a more stable flame. The geometric shape of the bluff body burner and the parameters affecting the combustion process are closely related to the aerodynamics of the circulation ficld. In particular, the structure of the circulation zone, and the positions of the front and back stagnation points are very helpful for understanding the process of flame stability.

Hedley and Jackson (1965) ${ }^{[6]}$ found that recirculation was helpful to the mixing of fuel and air. This finding led to universal attention to the formation of recirculation zone in the combustion system. Wingfield $(1967)^{[12]}$ conducted an experiment on double concentric jet with isothermal flow. He investigated the variations of the velocity field and flow intensity in the recirculation zone outside the combustion chamber under different bluff body thicknesses and velocity ratios. He concluded that the strongest mixing of the primary and secondary jets occurred when the bluff body thickness ratio $\left(d_{2} / d_{1}\right)$ is between 2 and 3 ; and that the intensity of the primary fuel jet decreased when the velocity ratio $\left(U_{s} / U_{p}\right)$ and momentum ratio $\left(G_{s} / G_{p}\right)$ increased. Focusing on the variations of double concentric jet in the combustion chamber with a cold flow, Habib and Whitelaw $(1979)^{[4,5]}$ measured the average velocity, turbulence intensity and Reynolds stress under different velocity ratios and number of swirls. They found that the length and velocity ratio of the outer recirculation zone were related to the geometric shape of the combustion chamber, and that the greater the velocity ratio, the greater the velocity gradient in the mixed zone. Smith et al $(1983)^{[10]}$ examined the distribution of static pressure, total stress, and velocity on the combustion chamber walls. They discovered that chemical reaction could change the size and position of the recirculation zone,

Received November, 1993. 


\begin{tabular}{|c|c|c|c|}
\hline & Nomenclature & & \\
\hline B.R. & blockage ratio $\left(d_{2} / d_{1}\right)^{2}$ & $U$ & mean velocity \\
\hline$d$ & diameter & $U_{p}$ & primary jet velocity \\
\hline$D_{e}$ & diameter of Combustion Chamber & $U_{s}$ & secondary jet velocity \\
\hline E.R. & expansion ratio $d_{s} / D_{c}$ & $\theta^{\prime}$ & modified Thring \& Newby circulation variable \\
\hline$G$ & momentum flux & $\alpha$ & cone angle \\
\hline$L_{\mathrm{rz}}$ & length of recirculation zone & & Subscript \\
\hline$P_{w}$ & wall static pressure & $o$ & single jet \\
\hline$P_{o}$ & static pressure of entrance wall & $p$ & primary jet \\
\hline$r_{e}^{\prime}$ & effective radius of double concentric jet & $s$ & secondary jet \\
\hline $\boldsymbol{R e}$ & Reynolds' number & cen & center \\
\hline
\end{tabular}

and that the static pressure distributions of both the combusting and cold flow were uneven. In the combusting flow, the static pressure increased; the total stress decreased; and the decline of the intensity and velocity both slowed down due to combustion reaction. Pan et al (1992) ${ }^{[7]}$ proposed five important parameters which may affect flame persistence: (1) blockage ratio of the bluff body; (2) cone angle (a) of the bluff body; (3) incoming mixture velocity (U); (4) turbulence intensity (I); (5) mixture equivalence ratio $(\phi)$. During burning, the recirculation zone behind the bluff body increases due to air expansion from burning, and the structure of the flow field also changes. Pan, Schmoll and Ballal (1992) ${ }^{[8]}$ studied the nature of turbulence of the cone-shaped flame in a closed flow field, measured the turbulence movement, Reynolds shear stress, the average axial stress gradient, and the structure of the recirculation zone. They found that the recirculation zone size decreased when the blockage ratio or turbulence intensity in the combusting flow increased. When the cone angle of the bluff body increased, the volume of the recirculation zone increased slightly. As the mixture equivalence ratio increased, the length of the recirculation zone decreased. When the turbulence intensity increased, the length of the recirculation zone distinctly decreased, whose value was close to the value in an isothermal flow field.

Chen, Driscoll, Kelly, Namajian and Scheffer $(1990)^{[1]}$ compared the effects of bluff body and eddy on flame stability. They presented two important parameters affecting the flame type: (1) eddy vortex circulation; (2) jet momentum. The square root of the flame length and fuel of these two types of flame and air momentum ratio, and the eddy vortex circulation are reciprocal. Both the center bluff body and eddy circulation can produce a large area of recirculation whose front stagnation point position is an important factor in determining flame stability and the delivery of energy to the nozzle. An improvement on the mixing of fuel and air can shorten the flame length considerably.

Schefer, Namazian and Kelly $(1978)^{[9]}$ measured the structure of the flow field behind the bluff body when methane was burned. They classified the flame into two types of jet that governs the circulation field: airHow or fuel-flow, which were determined by the velocity ratio between the primary fuel jet and the surrounding secondary air jet. They found that the secondary air jet tended to wrap around the center axis and form a tail circulation zone. The secondary air jet and the center primary fuel jet act on each other in the main circulation zone. Under non-combustion circumstances, there are obviously two stagnation points on the center axis line, which are respectively formed by the primary fuel jet and the surrounding secondary air jet. However, only one stagnation point exists on the center axis. The reason lies in that during combustion, the gas volume expands, and the density decreases, which increases the penetration of the fuel jet. This causes the stagnation point of the fuel jet to move downstream substantially. On the contrary, the stagnation point of the air jet is not much affected by combustion. Therefore, the stagnation points of air and fuel overlap. But the size of the total volume of the recirculation zone remains unaltered. This is different from another situation in which the recirculation zone increases considerably due to the expansion of burning air in the burning with premix flame.

Based on the similarity between the flow field of the projecting combustion chamber and the reverse gradient reattachment flow field, Eaton and Johnston $(1981)^{[3]}$ concluded that five major independent parameters may affect the reattachment length: (1) initial boundary layer state, (2) the initial boundary layer thickness, (3) freestream turbulence, (4) pressure gradient, (5) aspect ratio. Driver, Seegniller and Marvin (1987) ${ }^{[2]}$ analyzed the frequency spectrum of the wall surface pressure and velocity. They discovered that both had low frequency fluctuation which was generated by the fluctuation in the shear 
layer. As a result, the reattachment point also traveled back and forth in a low frequency. As for the circulation field structure, Troutt, Scheekleand Novanam (1984) ${ }^{[11]}$ measured the existence of a sizable eddy in the circulation division spot and reattachment zone. Yang, Yu (1983) ${ }^{[13]}$ measured the average velocity and turbulence intensity of the flow field of a projecting combustion chamber, and the wall surface pressure. They pointed out the presence of eddies in the recirculation zone. The surroundings of the reattachment point can be considered the high turbulence source. Large velocity gradients in the shear layer generate high turbulence which is propagated by diffusion and convection in and outside the recirculation zone. The flow field inside the recirculation zone appears anisotropic, but gradually turns isotropic after passing over the reattachment point.

\section{EXPERIMENTAL METHOD AND FACILI- TIES}

This experiments is to make observation and measurement of the cold flow field of the double concentric burner with a bluff-body. The purpose is to observe the variations of the aerodynamics inside the combustion chamber under different center bluff body blockage ratios, bluff body cone angles and velocity ratios. Fig.1 illustrates the systematic apparatus of the experiments.
The combustion chamber is built with transparent ackerlite to facilitate the injection of tracking agent for observing the aerodynamics in the combustion chamber. The inner radius of the combustion model is 29 $\mathrm{cm}$, and length $200 \mathrm{~cm}$, to ensure full development of downstream flow field in the combustion chamber. There are also some openings on the side of the chamber to insert the five-hole pitot tube for measuring. As far as the burner design is concerned, the influence of the recirculation is extremely crucial. This experiment mainly employs the helium bubbles method and the Tufts method to observe the recirculation zone behind the bluff body and the peripheral corner recirculation zone inside the projecting combustion chamber for the quantitative analysis of the flow field. The five-hole pitot tube is also used to measure the average velocity of the flow field, and the static pressure tap to measure the distribution of wall surface static pressure inside the combustion chamber. Fig.2 is the diagram of the geometric shape and size of the projecting combustion chamber. A blaster is used to deliver compressed air to the primary and secondary jets for simulating the fuel and air jets in an actual burner. A floating flow meter is used to measure the volume passing rate of the primary and secondary jets. Table 1 shows the major operations.

Helium bubbles are guided into the primary and secondary jets to simulate, respectively, the fuel and air jets in an actual burner. The center bluff body blockage ratio, bluff body cone angle and the velocity

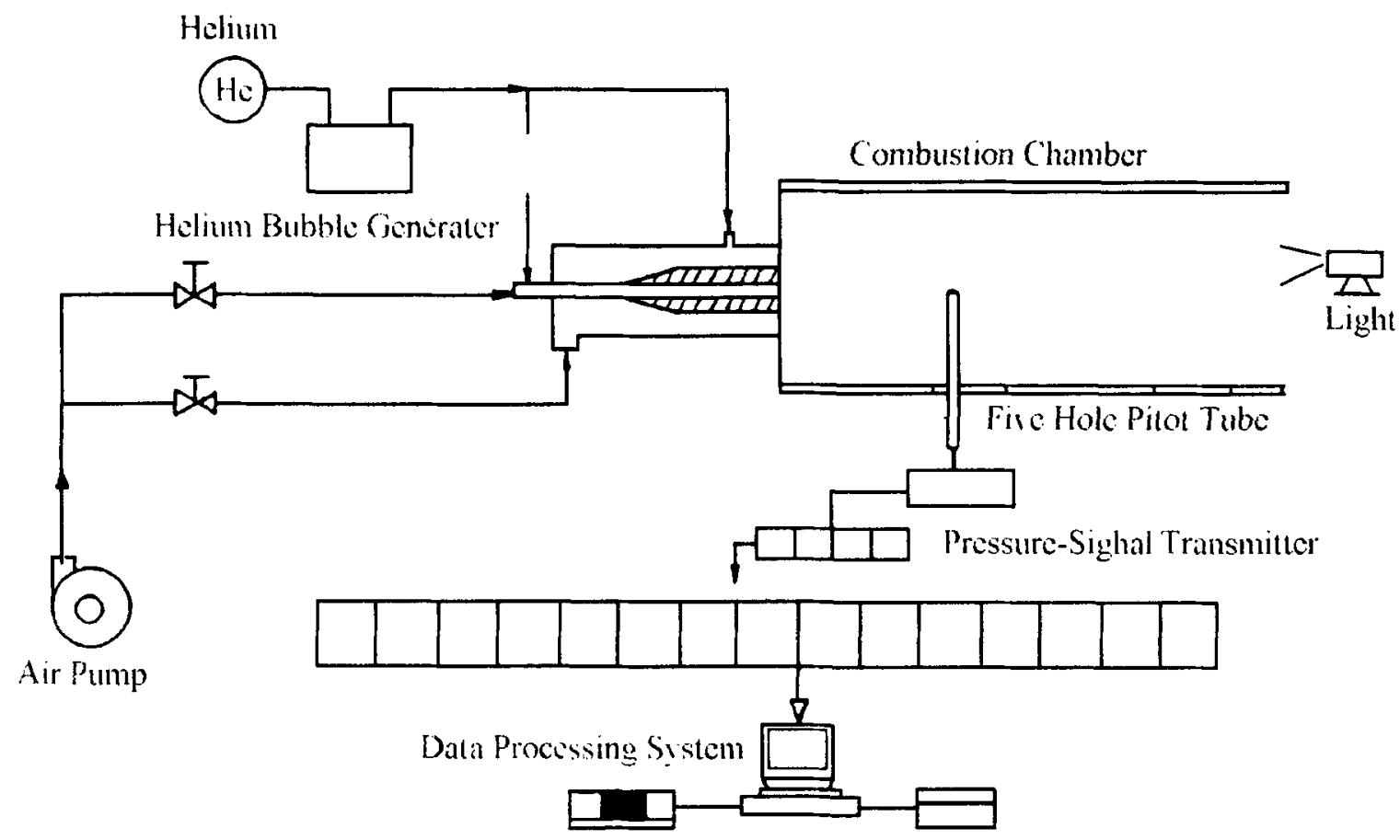

Fig.1 The systematic apparatus of the experiments 
ratio between the primary and secondary jets are changed to observe the variations of the aerodynamics in the recirculation zone behind the bluff body and within the combustion chamber by means of the Tufts method. An examination of the distribution of pressure inside the combustion chamber is helpful for understanding the circumstances of the flow field inside the combustion chamber and the velocity distribution. A pressure tap with an inner radius of $1 \mathrm{~mm}$ is installed on the wall of the combustion chamber, the other end of which is attached to a high accuracy pressure transducer to measure the variations of the wall surface pressure in the combustion chamber with the atmospheric pressure as reference. jet and the surrounding secondary air jet. When the primary jet emerges from the recirculation zone formed by the surrounding secondary jet, the center jet and the surrounding jet act on each other, which then leads to the emergence of an air-driven vortex and a fuel-driven vortex in the recirculation zone. The air-driven vortex is generated by the shear layer in the division of air flow around the edge of the bluff body and the fuel-drived vortex is generated by the center fuel jet. These two vortices turn in opposite directions. Besides, both their positions and structures change according to the changes in the momentum of the two jets. As a result, two stagnation points may appear on the symmetric axis at different times,

Table 1 Operation Condition

\begin{tabular}{|l|c|c|c|c|c|c|}
\hline Case & A & B & C & D & E & F \\
\hline$d_{1}(\mathrm{~mm})$ & 11 & 11 & 11 & 11 & 11 & 11 \\
\hline$d_{2}(\mathrm{~mm})$ & 21 & 33 & 44 & 33 & 33 & 33 \\
\hline$d_{s}(\mathrm{~mm})$ & 54 & 64 & 70 & 64 & 64 & 64 \\
\hline$\alpha$ & $0^{\circ}$ & $0^{\circ}$ & $0^{\circ}$ & $15^{\circ}$ & $30^{\circ}$ & $45^{\circ}$ \\
\hline$D_{e}(\mathrm{~mm})$ & 290 & 290 & 290 & 290 & 290 & 290 \\
\hline$B . R$. & 0.166 & 0.266 & 0.395 & 0.266 & 0.266 & 0.266 \\
\hline$E . R$. & 5.4 & 4.5 & 4.1 & 4.5 & 4.5 & 4.5 \\
\hline$U_{p}(\mathrm{~m} / \mathrm{s})$ & 14.0 & 14.0 & 14.0 & 14.0 & 14.0 & 14.0 \\
\hline$U_{s}(\mathrm{~m} / \mathrm{s})$ & 27.8 & 25.0 & 25.2 & 25.0 & 25.0 & 25.0 \\
\hline$R e_{p}$ & $1.05 \times 10^{4}$ & $1.05 \times 10^{4}$ & $1.05 \times 10^{4}$ & $1.05 \times 10^{4}$ & $1.05 \times 10^{4}$ & $1.05 \times 10^{4}$ \\
\hline$R e_{s}$ & $6.08 \times 10^{4}$ & $5.30 \times 10^{4}$ & $4.47 \times 10^{4}$ & $5.30 \times 10^{4}$ & $5.30 \times 10^{4}$ & $5.30 \times 10^{4}$ \\
\hline$G_{p}\left(\mathrm{Kg} \cdot \mathrm{m} / \mathrm{s}^{2}\right)$ & 0.02296 & 0.02296 & 0.02296 & 0.02296 & 0.02296 & 0.02296 \\
\hline$G_{s}\left(\mathrm{Kg} \cdot \mathrm{m} / \mathrm{s}^{2}\right)$ & 1.8142 & 1.8142 & 1.8142 & 1.8142 & 1.8142 & 1.8142 \\
\hline$G_{t}\left(\mathrm{Kg} \cdot \mathrm{m} / \mathrm{s}^{2}\right)$ & 1.8372 & 1.8372 & 1.8372 & 1.8372 & 1.8372 & 1.8372 \\
\hline$G_{s} / G_{p}$ & 79 & 79 & 79 & 79 & 79 & 79 \\
\hline$r_{e}^{\prime}$ & 2.51 & 2.785 & 2.768 & 2.785 & 2.785 & 2.785 \\
\hline$\theta^{\prime}$ & 0.173 & 0.192 & 0.191 & 0.192 & 0.192 & 0.192 \\
\hline
\end{tabular}

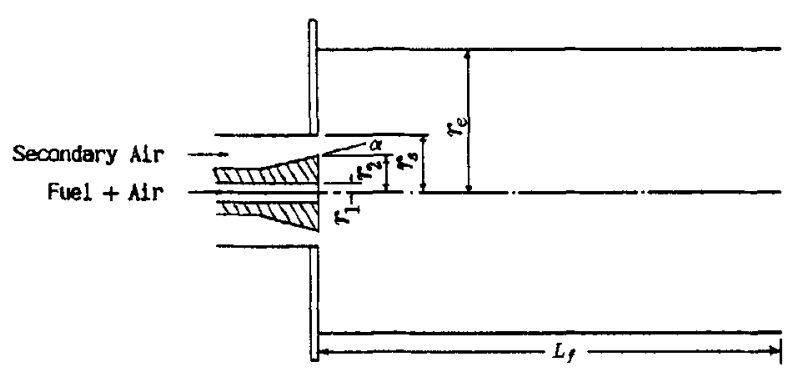

Fig.2 Schematic of combustor with a bluff-body

\section{RESULTS AND DISCUSSION}

The double concentric bluff-body jet can be regarded as the combination of the center primary fuel respectively caused by the center fuel jet and the surrounding air jet. The length of the recirculation zone $\left(L_{r z}\right)$ is defined as the distance between the fuel nozzle and the back stagnation point. The length may be affected by the bluff body diameter, bluff body cone angle and velocity.

As the fluid enters the projecting combustion chamber from the burner, the flow field is similar to the reverse gradient flow field. Fig. 3 is a typical reverse gradient flow field. Flow division occurs in the marginal layer when the secondary jet passes by the nozzle, which then generates the shear layer by working with the surrounding static fluid. The initial form of the shear layer is almost a straight line, unturning around the edge of the jet exit and parallel to the combustion chamber wall surface. Due to the difference be- 
tween the pressures from both sides of the shear layer, it curves toward the wall surface, and further downstream, the curve extends farther till it finally touches the wall surface. There is a very intense reverse gradient in this local flow field. Part of the fluid in the shear layer is absorbed into the recirculation zone somewhere in the upstream. Therefore, a recirculation zone is formed among the division point, shear layer and reattachment point. A corner eddy is also formed close to the projecting wall in the recirculation zone. After the fluid touches the wall surface, except part of it that flows upstream, most fluid still moves downstream to form another new boundary layer.

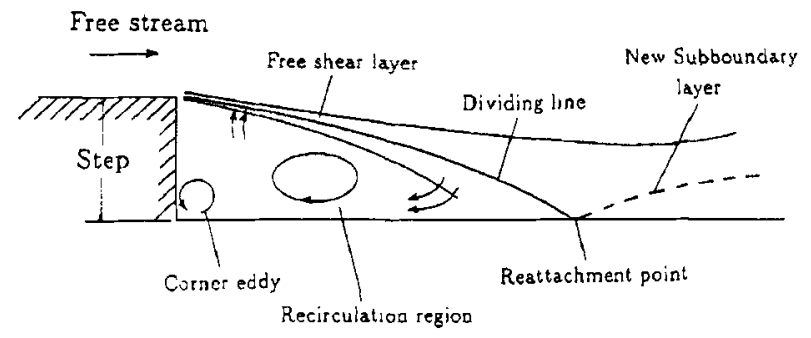

Fig.3 Schematic view of a typical reverse gradient flow field

\section{Observations of the Flow Fields with He- lium Bubbles}

The flow field of a double concentric burner mainly consists of the primary jet, the secondary jet and the recirculation zone. To distinguish the three parts, helium bubbles are introduced into the primary or secondary jets to serve as the tracking agent for observing the end flow changes behind the bluff body. Helium bubbles are injected into the primary and secondary jets to simulate the fuel and air nozzle in an actual burner. The recirculation zone observed behind the bluff body is not symmetric because the helium bubbles are injected into the jets with random speed and direction.

1.1 Effects of different blockage ratio of the center bluff-body

Fig.4 shows that two distinct eddies are formed in the recirculation zone as the helium bubbles from the primary jet are absorbed in the recirculation zone. These two eddies are the air-driven vortex and the fuel-driven vortex, with opposite turning directions. The variations in the bluff body blockage ratio in cases $\mathrm{A}, \mathrm{B}$ and $\mathrm{C}$ show that the greater the blockage ratio, the bigger the recirculation zone behind the bluff body. In Case A, the blockage ratio is small-0.166, and no distinct recirculation zone is present. As the blockage ratio increases to 0.266 in Case $\mathrm{B}$, a recirculation zone is generated behind the bluff body, but only appears periodically. When the blockage ratio increases to 0.395 in Case $\mathrm{C}$, a larger recirculation zone emerges behind the bluff body and stays there to form a triangle stable recirculation zone, with a length around $0.9 d_{2}$ to $1.2 d_{2}$. This length is similar to the length of $0.8 d_{2}$ to $1.5 d_{2}$ of the recirculation zone from a cylindrical flame persistence measured by Davies and Beer (1970) ${ }^{[14]}$. The fluid near the bluff body wraps around the center axis after leaving to nozzle, but the sideways fluid slides toward the com-
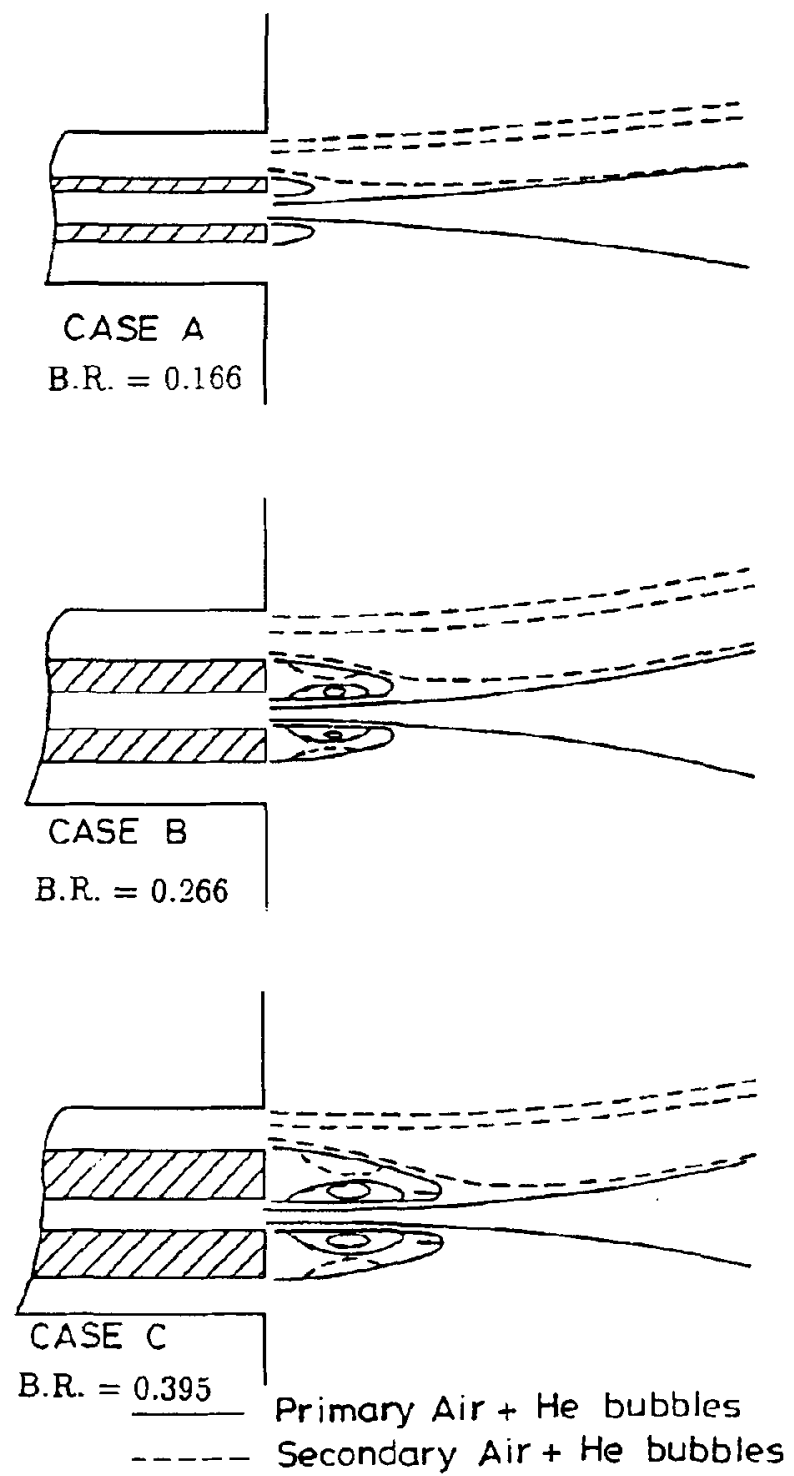

Fig. 4 The phenomenon of primary and secondary jets mixing under different blockage ratio

bustion chamber wall surface after leaving the nozzle to form an outer corner recirculation zone. It is very obvious that the formation of the recirculation zone 
is mainly affected by the size of the bluff body. Besides, the recirculation zone is formed by the primary fluid. The secondary fluid stays in the outer recirculation zone only. Therefore, the bluff body shape of a burner affects the shape and circulation intensity of the recirculation zone behind the bluff body, thus greatly affecting its effects on flame persistence.

\subsection{Effects of different bluff body cone angles}

This experiments used a cylindrical burner with variations in cone angle to observe the variations in the circulation fields. Helium bubbles are separately guided into the primary and the secondary jets to simulate the fuel and air jets in a burner. Fig. 5 shows the formation of the recirculation zone by helium bubbles from the primary jet when the cone angle of the center bluff body changes, but both the combustion chamber expansion ratio and the bluff body blockage ratio remain unchanged. In case $B$, with a cylindrical bluff body (baseline case), the recirculation zone behind the bluff body is of moderate size, with limited turbulence, and interrupted presence. When the bluff body changes to a cone, and the cone angle increases to 15 degrees, the recirculation zone behind the bluff body becomes larger and oval. When the angle increases, the length of the recirculation zone shortens, but the width increases. When helium bubbles emerge from the secondary jet, the bluff body cone angle causes the sliding angle of the fluid lines in the division by the bluff body edge to increase. As a result, the width of the recirculation zone increases, and the curvature increases, which leads to slight shortening of the recirculation length. The length of the recirculation zone is around $1.1 d_{2}$ to $1.3 d_{2}$, and the width around $1.1 d_{2}$ to $1.2 d_{2}$. Pan, Schmoll and Ballal $(1992)^{[8]}$ measured the length of the recirculation zone of a cylindrical bluff body to be around $1.5 d_{2}$, and width around $1.1 d_{2}$. They also noted that the recirculation zone consistently appeared behind the bluff body, and the helium bubbles from the primary jet caused considerable turbulence in it. This signifies that fuel would be absorbed into the recirculation zone at this point in time. Besides, high intensity of turbulence means better mixing of fuel and air, which makes it easier to achieve flame persistence.

\subsection{Effect of velocity ratio}

Helium bubbles are led into the primary and secondary jets for observing the changes in the recirculation field. In case $\mathrm{F}_{i}$ with a velocity ratio of 1.79 , the velocity of the secondary jet is greater than that of the primary jet which is thus brought upstream by the secondary jet. Therefore, the helium bubbles from

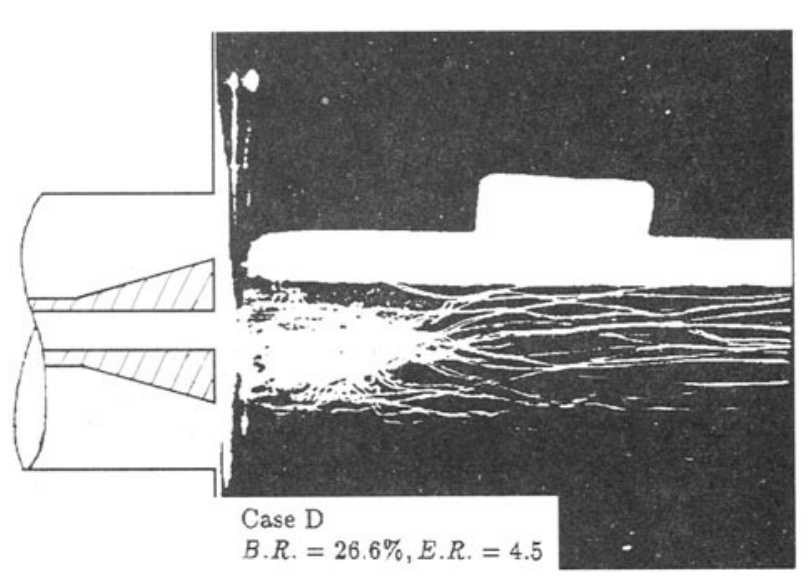

(a) Case D: $\alpha=15^{\circ}$

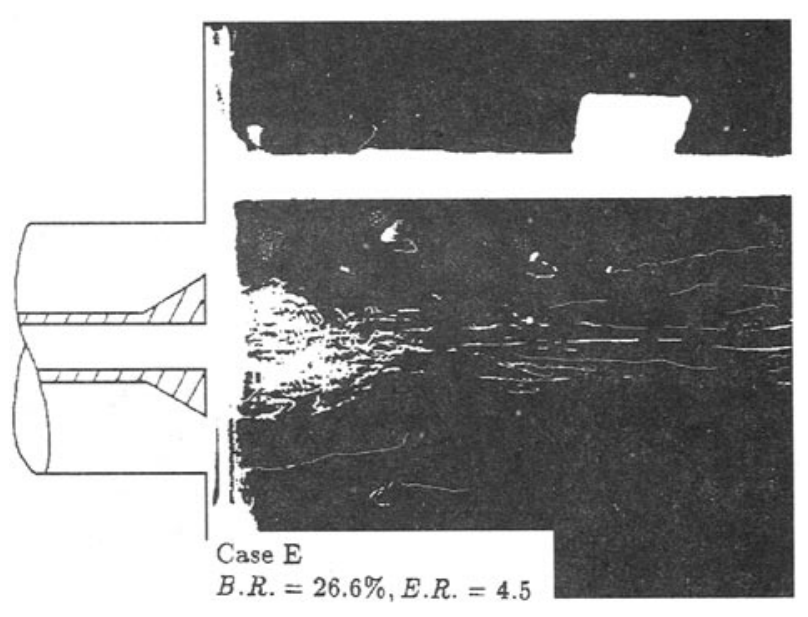

(b) Case E: $\alpha=30^{\circ}$

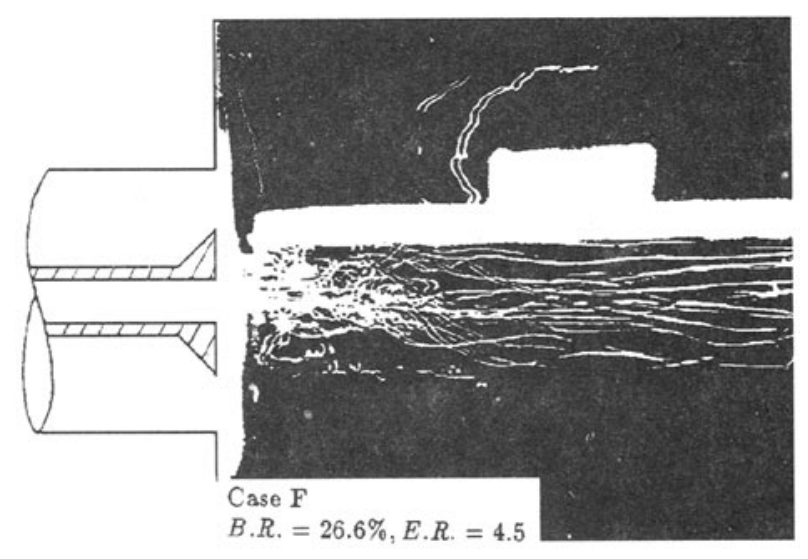

(c) Case F: $\alpha=45^{\circ}$

Fig.5 He-bubble tracinglines of primary jet around burner

the primary jet are absorbed into the recirculation zone. In Case G with a velocity ratio of 1.0 (Fig.6), the primary jet is not brought upstream, and the recirculation zone behind the bluff body thus disappear. 
In Case $\mathrm{H}$ where the velocity ratio further decreases to 0.56 , the velocity of the primary jet is greater than that of the secondary jet. The latter is then brought downstream by the former. As shown in Fig.6, helium bubbles are discharged into the secondary jet to simulate the air jet. We found that the helium bubbles from the secondary jets were absorbed into the recirculation zone.

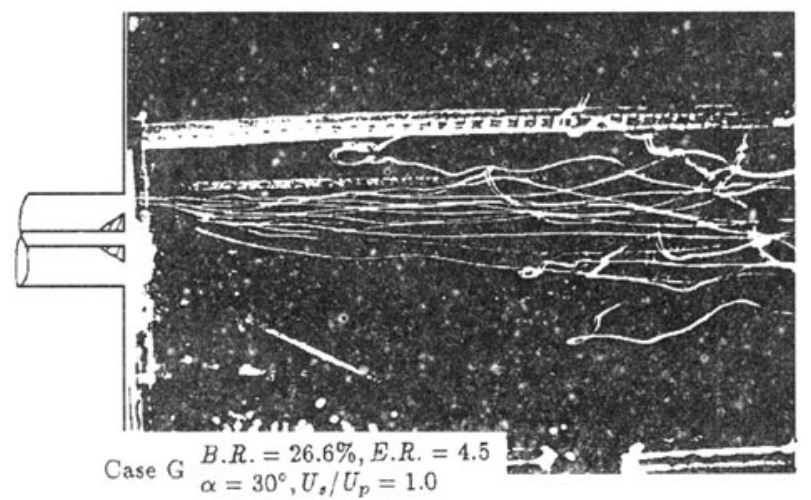

Fig.6 Photograph of the helium bubbles discharged into the secondary jet at $U_{s} / U_{p}=1.0$

Therefore, the velocities of the primary and secondary jets have considerable effects on the recirculation zone behind the bluff body. They determine whether the burning flame is controlled by the secondary air jet or the primary fuel jet. As shown in Fig.7, when helium bubbles are discharged into the secondary jet, if the bluff body con i angle is present, and the velocity of the primary jet is greater than that of the secondary jet, then the helium bubbles turn into an arc and quickly slide toward the center after leaving the nozzle. Therefore, if the bluff body blockage ratio is comparatively large, with the presence of a cone angle, and a substantial velocity ratio, the recirculation zone formed behind the bluff body is also large and with strong turbulence in it. This leads to

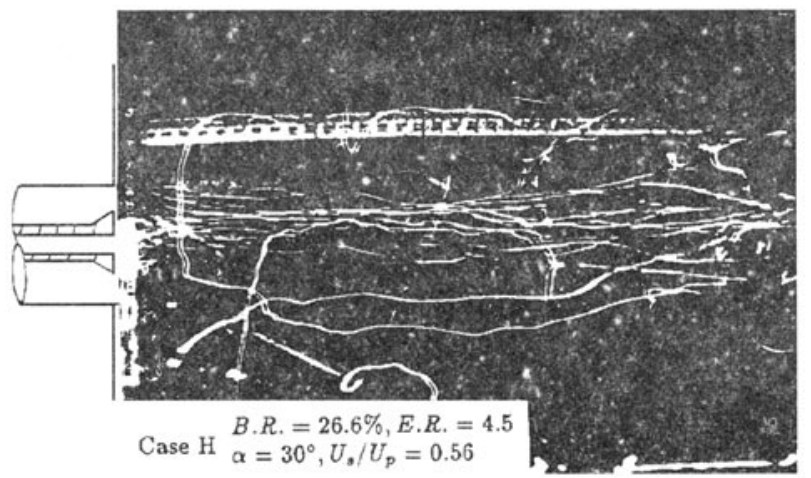

Fig.7 Photograph of the helium bubbles discharged into the secondary jet at $U_{s} / U_{p}=0.56$ better mixing of fuel and air, enabling the flame to remain stable instead of being blown away, thus achieving flame persistence. The recirculation zone disappears at velocity ratio of 1.0 . If the velocity ratio is smaller than 1.0, the secondary jet is absorbed into the recirculation zone behind the bluff body.

\section{Measured Results for the Center Axial Velocity}

2.1 Effects of different blockage ratios of the center bluff-body

Fig.8a shows when the secondary jet velocity is greater than the primary jet velocity, the center axial velocity immediately decreases after leaving the nozzle, and then rises again to its highest point. This can be explained as follows. The secondary jet forms a recirculation zone behind the bluff body after passing the bluff body. When the primary jet is discharged from the recirculation zone, it has to overcome the incoming fluid. As a result, the center axial velocity tends to rapidly decrease after the primary jet leaves the nozzle. The greater the velocity of the incoming fluid, the greater the decline of the primary jet's velocity. The primary and secondary jets near the nozzle can be seen as two separate jets. After the primary jet leaves the recirculation zone, it combines with the secondary jet to form a single jet. Therefore, the center axial velocity of the primary jet tends to decline to its lowest point, and then rises to the peak, which is the intermediate zone. Then the two jets join to form a single jet. After reaching the highest point, it gradually declines to form a mixed zone. In Case A, the velocity rises to its peak value at $0.6 D_{e}$, and then quickly declines to become stable around $2.5 D_{e}$. It attains full development around $x>3.5 D_{e}$. In the zone between $0.6 D_{e}$ and $2.5 D_{e}$, considerable variations in velocity gradients signify that fuel in the primary jet and air in the secondary jet are mainly mixed in this zone which is also the major combustion reaction zone of the flame. Our experiment reveals that the smaller the blockage ratio, the smaller the recirculation zone behind the bluff body near the nozzle, and the weaker the circulation. But there are greater variations of velocity gradients in the major mixing zone. On the other hand, though a greater blockage ratio creates a bigger recirculation zone behind the bluff body and stronger circulation, there are less variations in velocity gradients in the major mixing zone. These results are similar to those of an experiment by Wingfield $(1967)^{[12]}$ who found that the fastest mixing between the primary fuel jet and the secondary air jet occurred when the bluff body thickness ratio $d_{2} / d_{1}$ was between 2 to 3 . With different bluff body blockage ratios, constant primary jet velocity, and declining center axial 
velocity near the nozzle, two findings are concluded. First, the greater the bluff body blockage ratio, the greater the decline of the velocity, and the stronger the circulation in the recirculation zone. Second, the farther away from the nozzle the lowest velocity point is, the greater the recirculation zone. The size of the recirculation zone is the major factor affecting flame persistence and stability. Our measurement did not any stagnation point on the center axis, which signifies that the primary jet penetrates the recirculation zone, and fails to display any stagnation point on the center axis. This finding is consistent with the observation on helium bubbles. In Case A, the lowest velocity point is about $56 \%$ of the injected veiocity of the primary jet, which is located near $2.2 d_{1}$. In Case B, the lowest velocity point is about $36 \%$ of the injected velocity of the primary jet, which is located near $3.5 d_{1}$. In Case $C$, this point is about $20 \%$ of the injected velocity of the primary jet, which is located near $6.0 d_{1}$.

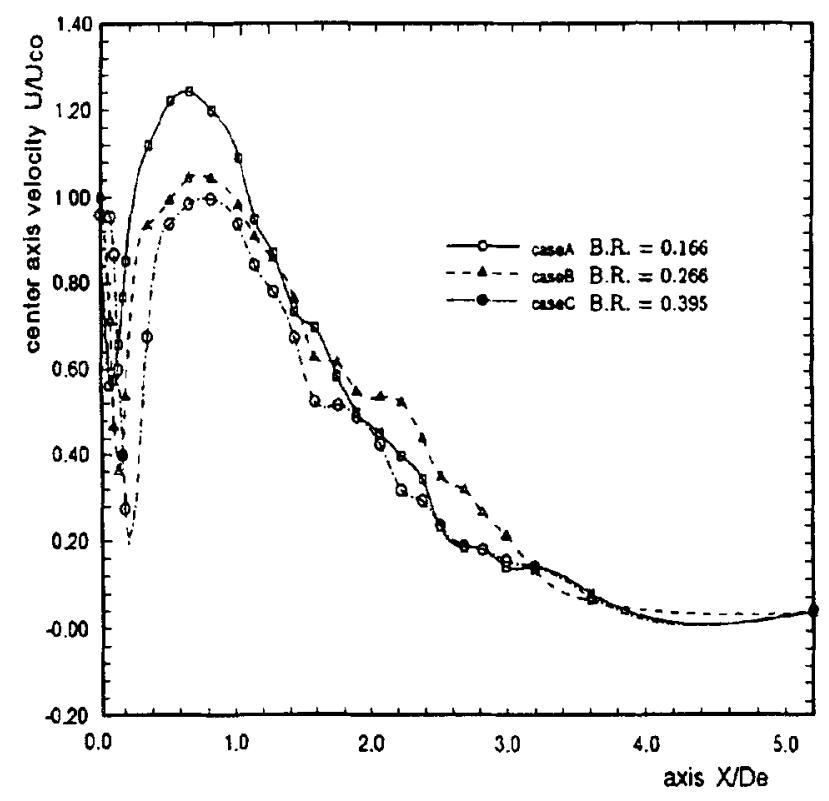

Fig.8a Center axial velocity under different blockage ratios

\subsection{Effects of different bluff-body cone angles}

Fig. $8 \mathrm{~b}$ is a comparison of the distribution of the center vertical velocity for different bluff body cone angles. We found that the variations in velocity gradients in locations farther from the main mixing zone's $0.6 D_{e}$ were all quite similar. But there are obvious differences near the nozzle where $X / D_{e}=0.2$. Fig.9 shows that the lowest velocity point of Case B $\left(\alpha=0^{\circ}\right)$ is about $36 \%$ of the exit velocity of the primary jet. The lowest velocity point of Case D $\left(\alpha=15^{\circ}\right)$ is about $28 \%$ of the exit velocity of the primary jet. The lowest velocity point of Case $\mathrm{E}$ $\left(\alpha=30^{\circ}\right)$ is about $12 \%$ of the exit velocity of the primary jet. There is negative velocity on the center axis in Case $F\left(\alpha=45^{\circ}\right)$. Therefore, there are two stagnation points with zero velocity on the center axis. The front and back stagnation points are caused respectively by the central primary jet and the surrounding secondary jet. part of the fluid in the secondary jet is absorbed back to upstream at the back stagnation point. It slides outward in a vertical direction after reaching the front stagnation point, and reacts with the sliding fluid from the primary jet. This phenomenon can be observed from the track of helium bubbles from the primary jet. This also demonstrates that an increase in the bluff body cone angle leads to an increase in the intensity of recirculation velocity in the recirculation zone behind the bluff body.

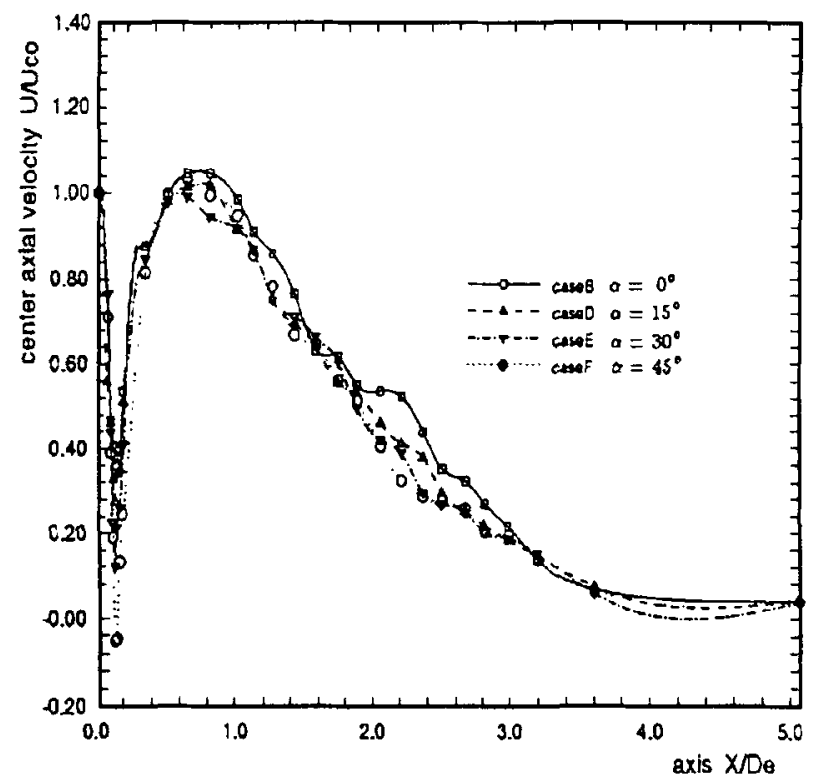

Fig.8b Center axial velocity under different cone angles

2.3 Effects of different velocity ratios of the secondary jet and primary jet

As shown in Fig.8c, in Case E, when the velocity of the secondary jet is greater than that of the primary jet, the center horizontal velocity of the primary jet immediately declines after leaving the nozzle, and then rises to the highest point, and finally gradually decreases. When the velocity of the secondary jet is equal to or smaller than that of the primary jet, the fluid of the primary jet immediately decreases after leaving the nozzle, without a decline followed by an increase. The reason is that the recirculation force of the recirculation zone formed by the secondary jet is not strong enough to counter-react the fluid from the primary jet. From Case $\mathrm{G}$ and $\mathrm{H}$, we found that all 
the areas with most gradient variations in velocity fall between $0.0 D_{e}$ and $0.2 D_{e}$. The changes gradually slow down after that. We can see from the velocity distribution near the nozzle in Fig.10c that in Case $\mathrm{G}\left(U_{s} / U_{p}=1.0\right)$, the velocity gradient is the greatest around $3 d_{1}<x<9 d_{1}$, and declines when $x>9 d_{1}$. In Case $\mathrm{H}$, at $U_{s} / U_{p}=0.56$, the velocity gradient is the greatest around $4.5 d_{1}<x<13 d_{1}$. The velocity variations become smaller in downstream areas. In Case $\mathrm{E}$ with greater velocity, there are larger average velocity gradients in the mixed areas.

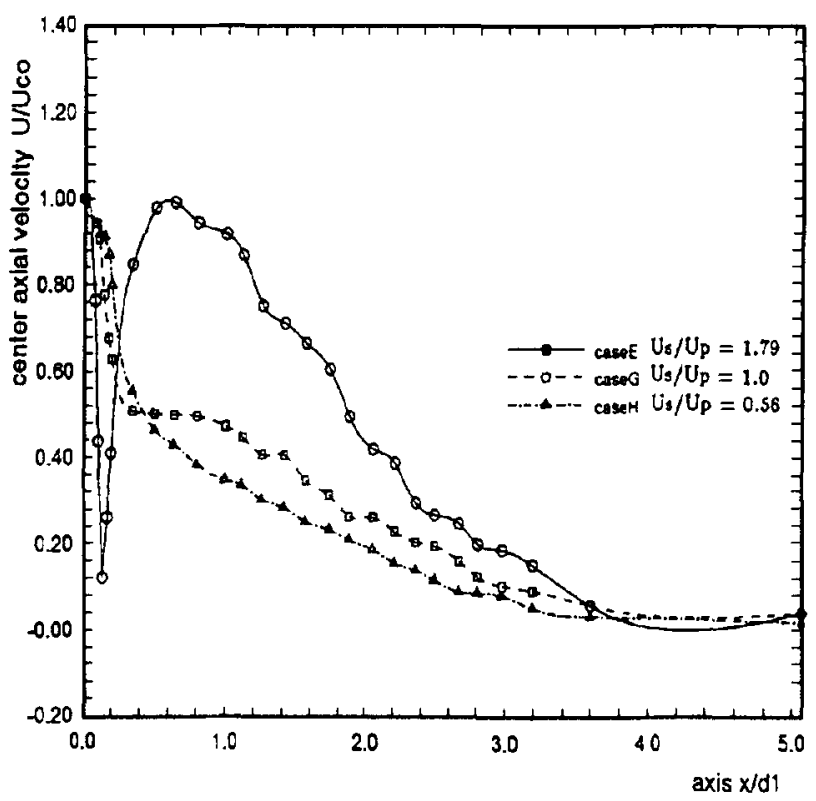

Fig.8c Center axial velocity under different $U_{s} / U_{p}$ ratios

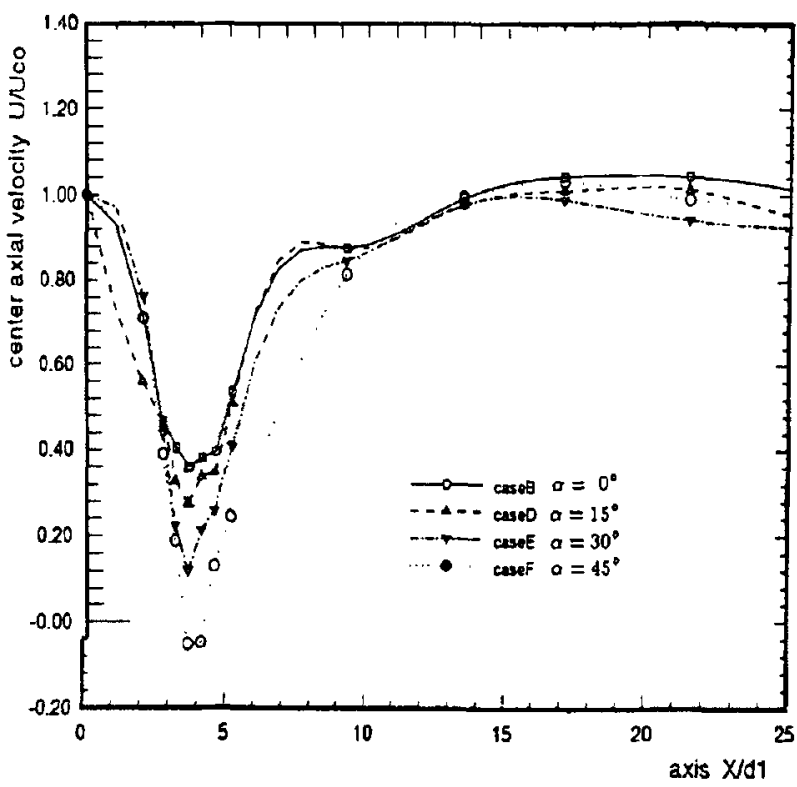

Fig.9 Center axial velocity near the Nozzle under different cone angles
3. Observation of the Corner Recirculation with Tufts and Distribution of Wall Surface Static Pressure

With Tufts observation, we found that as the bluff body cone angle increased, its reattachment length shortened slightly, and the area with greater recirculation velocity moved upstream. Fig.10 shows the distribution of wall surface static pressure when the bluff body angle of a burner changes, but the expansion ratio remains the same. When the bluff body cone angle increases, the area with the greatest reverse pressure gradient moves upstream. This is consistent with the results of Tufts observation mentioned earlier. Besides, the reattachment length also shortens, and the pressure gradients become greater in the reverse pressure zone.

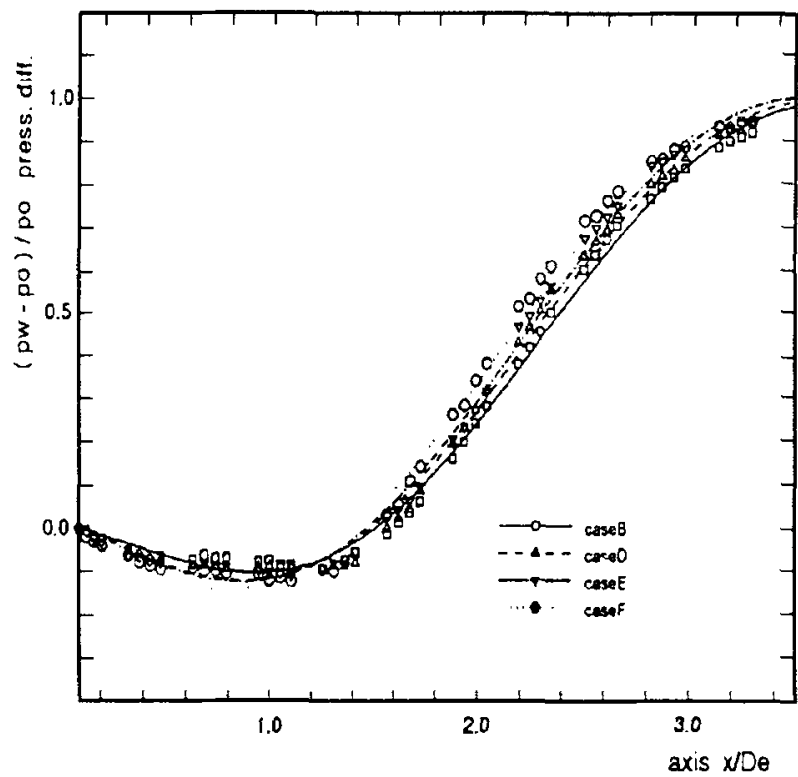

Fig.10 Wall pressure distribution under different cone angles

We can see in Fig.11 that in Case E, the pressure gradients are greater between $1.3 D_{e}$ and $2.9 D_{e}$. If the velocity ratio decreases to 1.0 as in Case $G$, the pressure gradients are greater between $1.4 D_{e}$ and 3.1 $D_{e}$. As the velocity ratio decreases further to 0.56 as in Case $\mathrm{H}$, the pressure gradients are greater between $1.5 D_{e}$ and $3.2 D_{e}$. It is obvious that as velocity ratio increases, the area with the greatest reverse pressure gradient moves further upstream. This shortens the reattachment length, and the reverse pressure gradient in the mentioned area increases. When velocity ratio changes, the position of the reattachment point is between $5.2 \mathrm{~h}$ and $6.7 \mathrm{~h}$. This is slightly different from the Tufts observation results. The area with a comparatively greater recirculation velocity is located in the reverse pressure zone. 


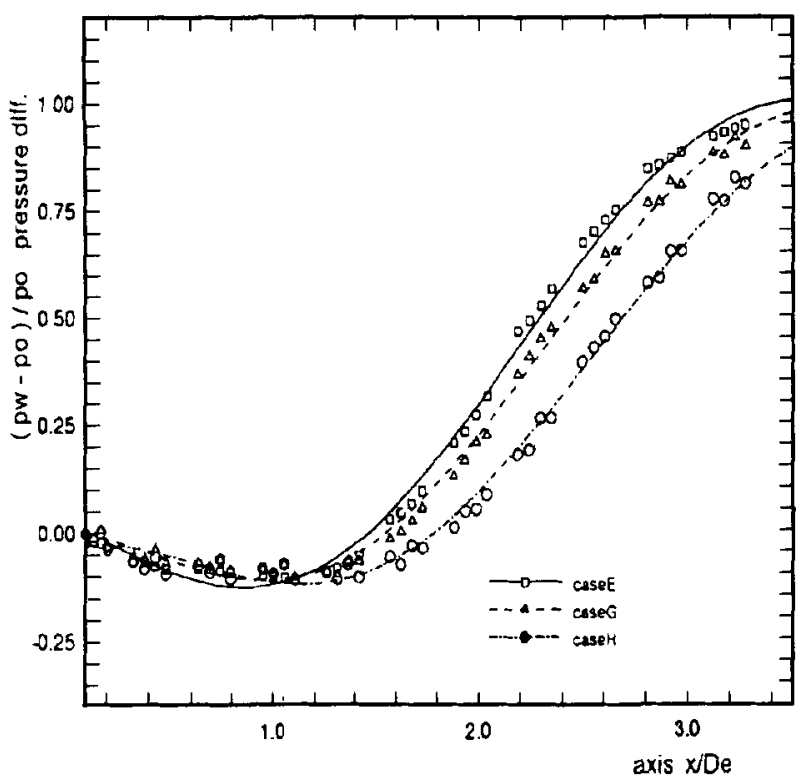

Fig.11 Wall pressure distribution under different values of $U_{s} / U_{p}$

\section{CONCLUSIONS}

1. There are two large structural eddies in the recirculation zone behind the bluff body, generated respectively by the primary fuel jet and the secondary air jet. The turning directions of these two eddies happen to be opposite. The value of the velocity ratio between the primary and secondary jets determines whether the circulation field is governed by the primary or the secondary jet. If the secondary jet controls the circulation field, the fluid from the primary jet will be brought into the recirculation zone. On the contrary, if the primary jet controls the circulation field, the fluid from the secondary jet will be absorbed into the recirculation zone.

2. The recirculation zone behind the bluff body is affected by the bluff body blockage ratio, bluff body cone angle and velocity ratio. The greater the bluff body blockage, the bigger the recirculation zone behind the bluff body. When a bluff body cone angle is present, the angle of the fluid passing by the edge of the bluff body increases, creating a larger recirculation zone. As the cone angle increases, there are only slight changes in the shape of the recirculation zone. But the recirculation intensity increases, and the turbulence within the recirculation zone turns strong.

3 . The smaller the blockage ratio and the greater the velocity ratio, the greater the changes in the center horizontal velocity gradients in the mixed area.
4. With proper bluff body blockage ratio, a greater bluff body cone angle and greater velocity ratio $\left(U_{s} / U_{p}\right)$ can lead to stronger turbulence in the recirculation zone formed behind the bluff body. This means better mixting of fuel and air, which makes it easier to achieve flame persistence.

\section{REFERENCES}

[1] Chen, R.H., Driscoll, J.F., Kelly, J., Namazian, M. and R.W. Schefer, "A Comparison of Bluff-Body and Swirl Stabilized Flames," Combustion Science and Technology, 71, pp. 197-217, (1990).

[2] Driver, D.M., Seegmiller, H.L., and J.G. Marvin, "Time Dependent Behavior of a Reattaching Shear Layer," AIAA J., 25, No.7, pp.914-919, (1987).

[3] Eaton, J.K., and J.P. Johnston, "A Review of Research on Subsonic Turbulent Flow Reattachment," AIAA J., 19, No.9, pp.1093-1100, (1981).

[4] Habib, M.A. and J.H. Whitelaw, "Velocity Characteristics of a Confined Coaxial Jet," (ASME) J. Fluids Engineering, 101, pp.521-529, (1979).

[5] Habib, M.A. and J.H. Whitelaw, "Velocity Characteristics of Confined Coaxial Jets with and Without Swirl," (ASME) J. Fluids Engineering, 102, pp.47-53, (1980).

[6] Hedley, A.B. and E.W. Jackson, "Recirculation and its Effects in Combustion Systems," J. Institute of Fuel, 38, pp.290-297, (1965).

[7] Pan, J.C., Vangsness, M.D. and D.R. Ballal, "Aerodynamics of Bluff-Body Stabilized Confined Turbulent Premixed Flames," (ASME) J. Engineering for Gas Turbines and Power, 114, pp.783-789, (1992).

[8] Pan, J.C., Schmoll, W.J. and D.R. Ballal, "Turbulent Combustion Properties Behind a Confined Conical Stabilizer," (ASME) J. Engineering for Gas Turbines and Power, 114, pp.33-38, (1992).

[9] Schefer, R.W., Namazian, M. and J. Kelly, "Velocity Measurements in a Turbulent Nonpremixed BluffBody Stabilized Flame," Combust. Sci. and Tech., 56, pp.101-138, (1987).

[10] Simth, G.D., Giel, T.V. and C.G. Catalano, "Measurements of Reactive Recirculating Jet Mixing in a Combustor," AIAA J., 21, No.2, pp.270-276, (1983).

[11] Troutt, T.R., Scheelke, B. and T.R. Norman, "Organized Structures in a Reattaching Separated Flow Field," J. Fluid Mech., 143, pp.413-427, (1984).

[12] Wingfield, G.J., "Mixing and Recirculation Pattern from Double Concentric Jet Burners Using and Isothermal Model," J. Institute of Fuel, pp.456-464, (1967).

[13] Yang, B.T. and M.H. Yu, "The Flowfield in a Suddenly Enlarged Combustion Chamber," AIAA J., 21, No.1, pp.92-97, (1983).

[14] Daview, T.W. and J.M. Beer, "Flow in the Wake of Bluff-Body Flame Stabilizers," 13th Symposium (International) on Combustion, pp.631-638, (1971). 